\title{
Autopsie des événements hydrométéorologiques extrêmes de 1969 en Tunisie
}

\author{
H. Boudhraâ ${ }^{1}$ and C. Cudennec ${ }^{2}$ \\ ${ }^{1}$ ESIER, Génie rural, Medjez el Bab, Tunisie \\ ${ }^{2}$ AGROCAMPUS OUEST, UMR1069, Sol Agro et hydrosystème Spatialisation, 35000 Rennes, France \\ Correspondence to: H. Boudhraâ (houdabr@gmail.com)
}

Received: 4 May 2015 - Accepted: 4 May 2015 - Published: 11 June 2015

\begin{abstract}
Résumé. Retrospective forensics of the 1969 extreme hydrometeorological events in Tunisia - Tunisia experienced extreme rainfall-flood events in September and October 1969 across most of the country. These events have been gauged and reconstituted in such a detailed manner that they are now the best known extreme events in Maghreb, both because they represent the most important events ever quantified and because many land uses and hydraulic facilities have changed and been set up since then, thus changing regimes. The retrospective forensics of these events are much valuable not only in terms of hydrological and geomorphological knowledge, but also in terms of actual and future water and infrastructure management as many dams, overexploited groundwater, human settlement and security stakes are now depending and vulnerable to such possible events in the future.
\end{abstract}

\section{Introduction}

L'autopsie (Forensics dans le vocable anglais) des catastrophes est une démarche scientifique qui permet de comprendre le déroulement des événements qui en sont à l'origine, les concours de circonstances et les facteurs aggravants, la rencontre entre aspects naturels et aspects anthropiques (IRDR, 2011). La quantification des phénomènes et l'analyse des contingences permettent de comprendre comment un événement est devenu extrême et catastrophique. L'analyse rétrospective d'événements réels passés permet de comprendre les possibles, mais la prospective au service de la sécurité doit généraliser les retours d'expérience et intégrer les changements pour diminuer les vulnérabilités, mieux se préparer à la gestion de crise, et gagner en résilience pour le futur (Cudennec et al., 2015). Cependant les événements extrêmes sont rares et les catastrophes du passé sont souvent mal documentées pour en tirer toutes les leçons.

Les événements hydrométéorologiques de 1969 en Tunisie ont été très intenses et dévastateurs. Le pays a été touché presque dans son ensemble. On déplorait à la date du 27 novembre 1969, 542 morts, 70540 maisons détruites et 303974 sans abris (Pias, 1970). Les implications hydrogéomorphologiques (Cudennec and de Lavenne, 2015) ont été très importantes (Pias, 1970; Stuckman, 1970; Claude and
Pontanier, 1971; Colombani et al., 1984). Ces événements sont caractéristiques d'un fonctionnement naturel à l'époque peu influencé par l'homme, avant l'intense aménagement structurel du territoire et des oueds des dernières décennies. Ces événements ont fait l'objet d'une documentation très riche en termes descriptifs, quantitatifs et réflexifs - documentation patrimoniale dont l'accessibilité est améliorée par la numérisation en accès libre en cours. La réanalyse de cette documentation permet de remettre en mémoire ces événements, dans une perspective de gestion des risques en Tunisie et plus largement au Maghreb, au sein de sa trajectoire de changement (Cudennec et al., 2007; Montanari et al., 2013). Cet article se concentre sur le volet hydrométéorologique, sa quantification, et les réflexions associées de l'époque.

\section{Les précipitations}

La Tunisie est soumise à des climats à dominante Méditerranéenne, caractérisée par la concentration de la pluviosité sur les mois froids de l'année, avec un été sec. Un étagement hydroclimatique et biogéographique s'organise du nord au sud du pays, sous influence synoptique saisonnière de différents secteurs de la mer Méditerranéenne et de l'océan Atlantique, et sous influence locale des reliefs (Slimani et al., 2007; Bac- 

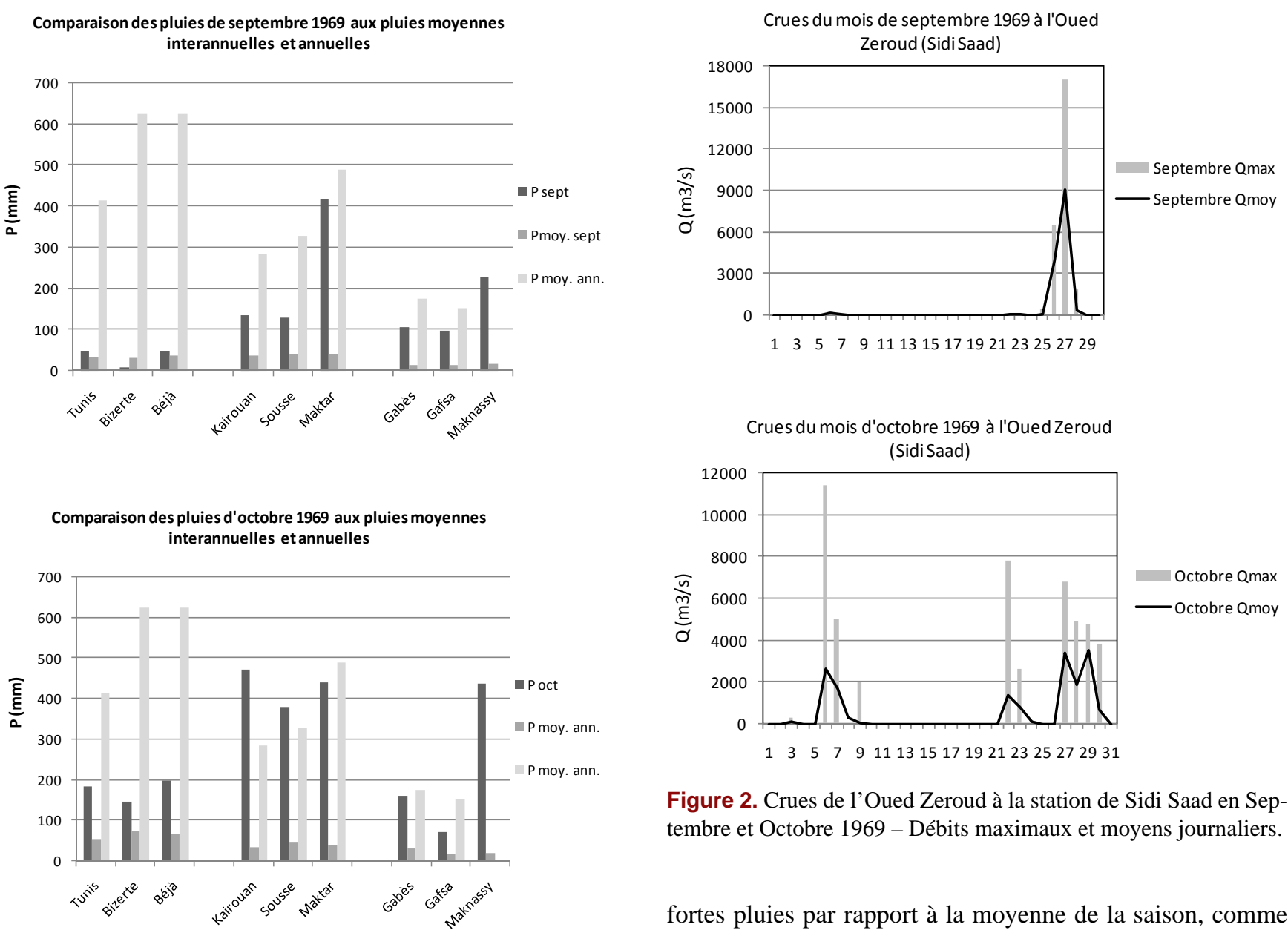

Figure 2. Crues de l'Oued Zeroud à la station de Sidi Saad en Septembre et Octobre 1969 - Débits maximaux et moyens journaliers.

Figure 1. Précipitations des mois de Septembre et d'Octobre 1969 comparées aux précipitations mensuelles moyennes sur 60 années et aux précipitations annuelles moyennes sur 50 années (Pias, 1970).

cour et al., 2012; Feki et al., 2012). Les événements météorologiques peuvent présenter de très fortes variabilités spatiotemporelles (Cudennec et al., 2005; Chargui et al., 2013).

Plusieurs épisodes pluvieux se sont succédés durant l'automne 1969, pendant 40 jours: 10 jours en Septembre qui a reçu plus de $400 \mathrm{~mm}$ dans certaines régions, et 30 jours en Octobre qui a reçu plus de $500 \mathrm{~mm}$ sur des surfaces importantes (Rodier, 1970). Ces pluies sont dues à un courant d'air froid polaire qui, dès qu'il a atteint le golfe de Gabès, a provoqué un courant de retour d'air sec Saharien. Au contact de la mer, ce dernier s'est humidifié et a subi une ascension rapide en altitude. Ce phénomène a donné naissance à des masses nuageuses très importantes, épaisses de $5000 \mathrm{~m}$ (Pias, 1970).

La troisième décade du mois de Septembre 1969 a été marquée par une grande pluviosité qui a touché presque tout le pays (Fig. 1). Seulement l'extrême Nord (gouvernorat de Bizerte) a connu des valeurs de pluie inférieures à la moyenne du mois. La région du sud Tunisien a connu les plus

fortes pluies par rapport à la moyenne de la saison, comme à Maknassy où les pluies ont atteint plus de 13 fois la valeur moyenne du mois de Septembre dans cette région.

Trois périodes très pluvieuses ont marqué le mois d'Octobre: du 3 au 8, du 11 au 17 et du 21 au 31. La première décade a connu une pluviométrie comprise entre 100 et $200 \mathrm{~mm}$ dans les parties centre et sud du pays (Sfax, Gabès et Djerba), avec 4 zones qui ont reçu plus de $200 \mathrm{~mm}$. La deuxième décade été beaucoup moins pluvieuse alors que la dernière décade était comparable à celle de Septembre avec des pointes de 200-300 mm et même $400 \mathrm{~mm}$ dans la partie centrale. La Fig. 2 présente quelques précipitations enregistrées en différentes régions de la Tunisie durant le mois d'Octobre 1969.

Les intensités de pluie ont été très variables dans le temps et dans l'espace. On a noté des intensités instantanées maximales de $80 \mathrm{~mm} \mathrm{~h}^{-1}$ en plusieurs endroits, mais aussi des pluies de faibles intensités et en continu, observées aux mêmes endroits et ailleurs, en alternance avec les pluies de fortes intensités. Si les fortes intensités ont eu une importance considérable sur les phénomènes d'érosion et l'arrivée des ondes des crues dévastatrices, les faibles intensités ont eu pour effet d'accentuer puis d'entretenir les inondations. 
Tableau 1. Caractérisation des débits de pointe et écoulements cumulés lors des événements de crues de l'automne 1969 dans les oueds principaux (Pias, 1970).

\begin{tabular}{llrrrl}
\hline Oued & Station & $\begin{array}{r}\text { Superficie } \\
\left(\mathrm{km}^{2}\right)\end{array}$ & $\begin{array}{r}\text { Débit } \\
\text { maximal } \\
\left(\mathrm{m}^{3} \mathrm{~s}^{-1}\right)\end{array}$ & $\begin{array}{r}\text { Volume } \\
\text { écoulé } \\
\left(10^{6} \mathrm{~m}^{3}\right)\end{array}$ & $\begin{array}{l}\text { Volume } \\
\text { écoulé } \\
\text { annuel moyen } \\
\left(10^{6} \mathrm{~m}^{3}\right)\end{array}$ \\
\hline Zeroud & Sidi Saad & 8950 & 18000 & 2000 & 90 \\
Merguellil & Haffouz & 793 & 4000 & 550 & \\
Kébir & Barrage & 250 & 2000 & & \\
Zigzaou & GP1 & 167 & 1500 & & \\
Nebhana & Barrage & 855 & 2200 & 155 & 34.1 \\
Bou Arada & Bou Arada & 95 & 600 & & \\
Tessa & Le Sers & 778 & 1800 & & \\
Tessa & Zouarine & 405 & 1250 & & \\
Mellegue & K13 & 9014 & 6000 & 680 & 15.7 \\
Tessa & Krib & 1100 & 1700 & & \\
Miliane & Cheylus & 1444 & 1300 & 255 & 28.5 \\
Medjerda & Medjez El Bab & 20930 & 1400 & 1150 & 1000 \\
\hline
\end{tabular}

Tableau 2. Caractéristiques hydrométéorologiques des événements de l'automne 1969 pour l'oued Zeroud à la station de Sidi Saad (Cruette et al., 1971).

\begin{tabular}{lcccl}
\hline & $\begin{array}{c}\text { Volume } \\
\text { écoulé } \\
\left(10^{6} \mathrm{~m}^{3}\right)\end{array}$ & $\begin{array}{c}\text { Lame } \\
\text { ruisselée } \\
(\mathrm{mm})\end{array}$ & $\begin{array}{c}\text { Pluie } \\
\text { moyenne } \\
(\mathrm{mm})\end{array}$ & $\begin{array}{l}\text { Coefficient de } \\
\text { ruissellement } \\
(\%)\end{array}$ \\
\hline Troisième décade de septembre & 1184 & 133 & 230 & 58 \\
Septembre & 1203 & 134 & 278 & 48 \\
Octobre & 1433 & 160 & 400 & 40 \\
Total Septembre-Octobre & 2636 & 294 & 678 & 43.2 \\
\hline
\end{tabular}

\section{Les crues}

Les crues des oueds Tunisiens peuvent être très brutales et intenses (Rodier, 1970), ce qui implique trois grandes difficultés métrologiques (Cruette et al., 1971):

- il faut être sur place au moment de la pointe de crue;

- la mesure des fortes vitesses (plus de $5 \mathrm{~m} \mathrm{~s}^{-1}$ ) est presque impossible;

- les rivières sont souvent caractérisées par des lits mobiles, ce qui rend difficile la connaissance du profil en travers au moment du pic.

Les événements de 1969 ont fait l'objet de mesures directes et d'estimations ex-post très précieuses. Le Tableau 1 présente les débits maximaux enregistrés durant l'automne 1969 sur les plus importants oueds de la Tunisie ainsi que les écoulements cumulés. La comparaison des apports de crues enregistrés en Septembre et Octobre 1969, aux apports moyens annuels des principaux oueds montre bien les énormes quantités d'eau écoulées dans cette période. Il a été estimé que 3 à 4 milliards de $\mathrm{m}^{3}$ d'eau se sont écoulés vers la mer (Pias, 1970).
L'écoulement de l'oued Zeroud à la station de Sidi Saad a été particulièrement étudié par les hydrologues de l'ORSTOM (France) et de la DRH (Tunisie). Des mesures des débits ont été effectuées pendant toutes les périodes des crues de l'automne 1969 (Fig. 2). Ces mesures ont montré que, sous certaines conditions favorables, il est possible de recueillir sur le terrain des éléments de calcul de la crue, même en situation extrême (Cruette et al., 1971). Des mesures des hauteurs d'eau, des mesures régulières de débits, et des mesures de vitesse au flotteur ont ainsi été réalisées, et le profil en travers et les variations du fond du lit de l'oued ont été décrits. Il a été noté que la crue du 27 Septembre 1969 a atteint un débit spécifique maximal de $1910 \mathrm{~L} \mathrm{~s}^{-1} \mathrm{~km}^{2}$. Pour un bassin de $8950 \mathrm{~km}^{-2}$, cela paraît être un record pour l'Afrique du Nord. En ce qui concerne la période de retour, depuis 1870 seulement deux crues ont présenté des débits du même ordre de grandeur, en 1904 et 1931. Ceci a conduit à estimer que la crue de 1969 est cinquantennale (Cruette et al., 1971).

Sur la base des mesures, les hydrologues ont pu calculer et estimer plusieurs indicateurs sur les crues de l'automne 1969. Ainsi, les débits maximaux ont été identifiés, allant de 250 à $17050 \mathrm{~m}^{3} \mathrm{~s}^{-1}$. Les volumes écoulés, calculés à la main à 
l'époque, ont été estimés à des valeurs allant de $10.7 \times 10^{6}$ à $1170 \times 10^{6} \mathrm{~m}^{3}$. Une estimation du transport solide de l'ordre de $275 \times 10^{6} \mathrm{~m}^{3}$ a également été donnée, ainsi que quelques indications sur les coefficients de ruissellement (Tableau 2).

\section{Retour d'expérience d'époque}

Ces événements extrêmes, et les enjeux de leur caractérisation hydrométéorologique vis-à-vis de la sécurité des biens et des personnes d'une part, de leur potentiel de mobilisation en termes de ressources en eau d'autre part, ont conduit à l'époque à un retour d'expérience approfondi. Cette réflexivité a fortement orienté la stratégie hydrométrique et la politique nationale d'aménagement hydraulique des décennies suivantes (Kallel and Colombani, 1974; Kallel et al., 1975); et mérite d'être rappelée aujourd'hui, à l'heure de l'optimisation des réseaux d'observation, de la modernisation des méthodes de mesure, et du développement des capacités et des compétences des services et des agents opérationnels. Au delà des remarquables technicité et conscience professionnelle des opérateurs de terrain, les enseignements métrologiques suivants ont été tirés (Cruette et al., 1971):

1. Si les rives de l'oued sont instables, il est préférable de chercher un autre site, car on ne peut pas utiliser d'autres méthodes que la méthode chimique si la station elle-même se déplace. Or, cette méthode n'est applicable que pour des débits inférieurs à $3000 \mathrm{~m}^{3} \mathrm{~s}^{-1}$.

2. Pendant les périodes de crue, il faut la présence continue d'un agent hydrométriste capable d'exécuter un jaugeage au téléphérique en période de crue moyenne avec des vitesses atteignant au moins $3 \mathrm{~m} \mathrm{~s}^{-1}$. Cet agent, capable de prendre des initiatives, doit avoir cependant des consignes précises sur ce qu'il convient de faire.

3. Un hydrométriste particulièrement compétent et un ingénieur hydrologue confirmé doivent assister à une partie des crues au moins.

4. La station doit être équipée d'un téléphérique solide et bien conçu susceptible d'utiliser un saumon de $100 \mathrm{~kg}$ et, si possible, de deux limnigraphes et d'une échelle facile à suivre.

5. En période de très forte crue, l'observateur doit suivre régulièrement les niveaux sur l'échelle; il doit avoir des consignes pour recueillir les éléments nécessaires en vue de déterminer la hauteur lorsqu'il y a des vagues de $1 \mathrm{~m}$ et plus.

6. Toutes les dispositions doivent être prises pour faire des mesures de vitesse aux flotteurs à partir des arbres charriés en prenant le câble du téléphérique pour extrémité amont ou aval de la base de mesure, le chariot étant mis au milieu permet de repérer la position des flotteurs dans le profil en travers. Ne pas oublier de prendre des flotteurs au voisinage des deux berges. Des consignes précises seront données aux hydrométristes sur ce point. Bien matérialiser la base de mesure.

7. A la décrue, dès que la vitesse descend en-dessous de 3 ou $4 \mathrm{~m} \mathrm{~s}^{-1}$, il faut procéder à des jaugeages réguliers. La connaissance du profil en travers est prioritaire.

8. Il faut faire fréquemment des jaugeages de basses eaux de façon à avoir débits et cotes du fond avant et après la crue.

9. Il faut faire un lever régulier avec sondage si nécessaire du profil en travers rocheux.

10. Si le rocher est trop profond, il faut employer le dispositif des piles de briques pour obtenir la profondeur maximale a posteriori.

11. Lorsqu'un hydrométriste très expérimenté ou un ingénieur hydrologue confirmé est là, on peut tenter un profil en travers avec le saumon nu. La méthode demande d'ailleurs à être perfectionnée. Il faut dissocier dans l'esprit des hydrologues et hydrométristes les mesures de vitesses et de profondeurs.

12. Une liaison radio permanente est extrêmement utile; elle permet, outre les avantages signalés plus haut, de supprimer l'impression d'isolement qui n'est pas sans influencer les exécutants aux moments les plus difficiles.

\section{Références}

Baccour, H., Slimani, M., and Cudennec, C.: Etude synoptique conjointe des structures spatiales de l'évapotranspiration et de variables climatiques corrélées en Tunisie, Hydrolog. Sci. J., 57, 818-829, 2012.

Chargui, S., Slimani, M., and Cudennec, C.: Statistical distribution of rainy events characteristics and instantaneous hyetographs generation (Merguellil watershed in central Tunisia), Arab. J. Geosci., 6, 1581-1590, 2013.

Claude, J. and Pontanier, R.: Quelques données sur les crues, les modifications morphologiques, et l'évolution des eaux et des alluvions du bassin Leben - Ouadrane après les pluies de l'automne 1969, République Tunisienne, Service hydrologique, Tunis, p. 41, 1971.

Colombani, J., Olivry, J. C., and Kallel, R.: Phénomènes exceptionnels d'érosion et de transport solide en Afrique aride et semiaride, IAHS Publ. 144, 295-300, 1984.

Cruette, J., Rodier, J. A., Dubée, G., and Gualde, R.: L'Oued Zeroud, mesures de débits pendant les crues exceptionnelles de l'automne 1969, Avril 1971, Rapport du service hydrologique de l'ORSTOM et de la DRH, Service hydrologique, Tunis, p. 42, 1971.

Cudennec, C. and de Lavenne, A." Hydrogeomorphology - A longterm scientific interface, Hydrol. Res., 46, 175-179, 2015. 
Cudennec, C., Slimani, M., and Le Goulven, P.: Accounting for sparsely observed rainfall space-time variability in a rainfallrunoff model of a semiarid Tunisian basin, Hydrolog Sci. J., 50, 617-630, 2005.

Cudennec, C., Leduc, C., and Koutsoyiannis, D.: Dryland hydrology in Mediterranean regions - a review, Hydrolog. Sci. J., 52, 1077-1087, 2007.

Cudennec, C., Demuth, S., Mishra, A., and Young, G.: Hydrological Sciences and Water Security: Past, Present and Future, PIAHS, 366, p. 201, 2015.

Feki, H., Slimani, M., and Cudennec, C.: Incorporating elevation in rainfall interpolation in Tunisia using geostatistical methods, Hydrolog. Sci. J., 57, 1294-1314, 2012.

IRDR: Forensic investigations of disasters: The FORIN Project, RDRD FORIN Publ. No. 1, Beijing, p. 28, 2011.

Kallel, M. R. and Colombani, J.: Aperçu sommaire sur les caractéristiques des principaux bassins versants du secteur Nord et Ichkeul, plan directeur de lutte contre les inondations, Direction des Ressources en Eau, Tunis, p. 11, 1974.

Kallel, M. R., Bouzaiane, S., Ghorbel, A., Boche Duval, J. M., Claude, J., Gouyet, R. C., and Lamachère, J. M.: Etude hydrologique préliminaire des Oueds Zeroud et Merguellil, Rapport de la DRE et l'ORSTOM, Service hydrologique, Tunis, p. 402, 1975.
Montanari, A., Young, G., Savenije, H., Hughes, D., Wagener, T., Ren, L., Koutsoyiannis, D., Cudennec, C., Grimaldi, S., Blöschl, G., Sivapalan, M., Beven, K., Gupta, H., Arheimer, B., Huang, Y., Schumann, A., Post, D., Taniguchi, M., Boegh, E., Hubert, P., Harman, C., Thompson, S., Rogger, M., Hipsey, M., Toth, E., Viglione, A., Di Baldassarre, G., Schaefli, B., McMillan, H., Schymanski, S., Characklis, G., Yu, B., Pang, Z., and Belyaev, V.: "Panta Rhei - Everything Flows": Change in hydrology and society - The IAHS Scientific Decade 2013-2022, Hydrolog. Sci. J., 58, 1256-1275, 2013.

Pias, J.: Les inondations de septembre et octobre 1969 en Tunisie, Partie I: Etude pédologique, rapport technique de l'UNESCO, UNESCO, Paris, p. 36, 1970.

Rodier, J.: Crues exceptionnelles récentes en Tunisie et à Madagascar, Rapport du Comité National de Géographie, Commission d'hydrologie fluviale, Paris, p. 5, 1970.

Slimani, M., Cudennec, C., and Feki, H.: Structure du gradient pluviométrique de la transition Méditerranée-Sahara en Tunisie: déterminants géographiques et saisonnalité, Hydrolog. Sci. J., 52, 1088-1102, 2007.

Stuckmann, G.: Les inondations de septembre et octobre 1969 en Tunisie, Partie II: Les effets morphologiques des inondations de septembre-octobre 1969 dans le centre et le sud de la Tunisie, UNESCO, Paris, p. 35, 1970. 